Accepted August 30, 2014

Preprint typeset using LTEX style emulateapj v. 12/16/11

\title{
THE VERY FAINT END OF THE UV LUMINOSITY FUNCTION OVER COSMIC TIME: CONSTRAINTS FROM THE LOCAL GROUP FOSSIL RECORD
}

\author{
DANiEl R. Weisz ${ }^{1,2,4}$, Benjamin D. JOHNSON ${ }^{1}$, ChARlie CONROY ${ }^{3,1}$ \\ Accepted August 30, 2014
}

\begin{abstract}
We present a new technique to estimate the evolution of the very faint end of the UV luminosity function (LF) out to $z \sim 5$. Measured star formation histories (SFHs) from the fossil record of Local Group galaxies are used to reconstruct the LF down to $M_{\mathrm{UV}} \sim-5$ at $z \sim 5$ and $M_{\mathrm{UV}} \sim-1.5$ at $z<1$. Such faint limits are well beyond the current observational limits and are likely to remain beyond the limits of next generation facilities. The reconstructed LFs, when combined with direct measurements of the LFs at higher luminosity, are well-fit by a standard Schechter function with no evidence of a break to the faintest limits probed by this technique. The derived faint end slope, $\alpha$, steepens from $\approx-1.2$ at $z<1$ to $\approx-1.6$ at $4<z<5$. We test the effects of burstiness in the SFHs and find the recovered LFs to be only modestly affected. Incompleteness corrections for the faintest Local Group galaxies and the (unlikely) possibility of significant luminosity-dependent destruction of dwarf galaxies between high redshift and the present epoch are important uncertainties. These and other uncertainties can be mitigated with more detailed modeling and future observations. The reconstructed faint end LF from the fossil record can therefore be a powerful and complementary probe of the high redshift faint galaxies believed to play a key role in the reionization of the Universe.

Keywords: galaxies: evolution — galaxies: high-redshift — Local Group — galaxies: luminosity function, mass function
\end{abstract}

\section{INTRODUCTION}

Knowledge of the faint end of the galaxy LF is essential for understanding many aspects of the high redshift universe including the extent to which faint galaxies contribute to the total cosmic SFR density, to the reionization of the universe, and to gamma ray burst rates. The deepest Hubble Space Telescope (HST) images are "only" capable of reaching absolute magnitudes of $M_{\mathrm{UV}} \sim-17,{ }^{5}$ at $z \sim 5$ (Bouwens et al. 2014). However, several phenomena require knowledge of the UV LF to much fainter limits. For example, models that are able to simultaneously reionize the universe with galaxies by $z \sim 6$ and match other constrains such as the Thomson optical depth inferred from the cosmic microwave background must extrapolate the UV LF to $M_{\mathrm{UV}} \gtrsim-10$ (for nominal assumptions regarding the escape fraction of ionizing photons, the topology of the IGM, etc.; see, e.g., Dijkstra et al. 2004; Kuhlen \& Faucher-Giguère 2012; Robertson et al. 2013; Boylan-Kolchin et al. 2014). Even the James Webb Space Telescope (JWST) may not reach such faint limits (Windhorst et al. 2006), suggesting that direct detection of the faint galaxies thought to be key for reionization will prove elusive for decades.

Here, we present a new technique capable of probing the rest-frame UV properties of very faint galaxies across cosmic time. Using star formation histories (SFHs) of Local Group (LG) dwarf galaxies measured from the analysis of deep color magnitude diagrams (CMDs), we are able to synthesize the

\footnotetext{
${ }^{1}$ Department of Astronomy, University of California at Santa Cruz, 1156 High Street, Santa Cruz, CA, 95064; drw@ucsc.edu

${ }^{2}$ Astronomy Department, Box 351580, University of Washington, Seattle, WA, USA

${ }^{3}$ Harvard-Smithsonian Center for Astrophysics, 60 Garden St., Cambridge MA 02138 , USA

${ }^{4}$ Hubble Fellow

5 Throughout this paper we assume AB magnitudes and use "UV" as shorthand for the GALEX FUV bandpass, which has a central wavelength of $1550 \AA$
}

UV luminosity evolution of faint galaxies to high redshifts. With this approach we can estimate the UV LF to limiting magnitudes of $M_{\mathrm{UV}} \sim-5$ at $z \sim 5$ and -1.5 at $z<1$.

\section{METHODOLOGY}

The primary data used in this analysis are the SFHs of 37 LG dwarf galaxies that were measured by modeling their HST/WFPC2-based resolved star CMDs (Weisz et al. 2014). UV luminosities as a function of redshift are then constructed by coupling the SFHs to the Flexible Stellar Population Synthesis code (FSPS; Conroy et al. 2009, 2010). Once various sources of incompleteness are included, we fit a Schechter function (Schechter 1976) to a combination of these reconstructed LG LFs and more luminous literature values. Here, we describe this procedure in detail, including the assumptions and uncertainties in our analysis.

\subsection{Star Formation Histories of Local Group Dwarfs}

Our sample consists of 37 dwarfs galaxies located within the LG ( $\sim 50 \%$ of the known LG dwarf population). Sample galaxies have low present-day metallicities $\left(Z \lesssim 0.2 Z_{\odot}\right)$, stellar masses $\left(10^{4} \lesssim M_{\star} / M_{\odot} \lesssim 10^{8}\right)$, and dust content $\left(\mathrm{A}_{\mathrm{V}} \lesssim 0.5\right.$; Dolphin et al. 2003). Our sample contains systems of all morphological types and environments (Milky Way and M31 satellites, isolated systems) found in the LG, including 'ultrafaint' dwarfs. Overall, our sample is broadly representative of the entire LG dwarf galaxy population.

SFHs of these galaxies are presented in Weisz et al. (2014). They were measured by modeling CMDs from archival HST/WFPC2 imaging using the maximum likelihood CMD fitting package MATCH (Dolphin 2002). An example SFH derived from CMD fitting is shown in Figure 1. Full details of MATCH and application to the LG sample can be found in Dolphin (2002) and Weisz et al. (2014), respectively. Important for this study is the fact that the SFH time resolution is, at best, $\sim 10-15 \%$ of the lookback age. Thus, the highest well-resolved redshift is $z \sim 5$. This is a fairly stringent limit 

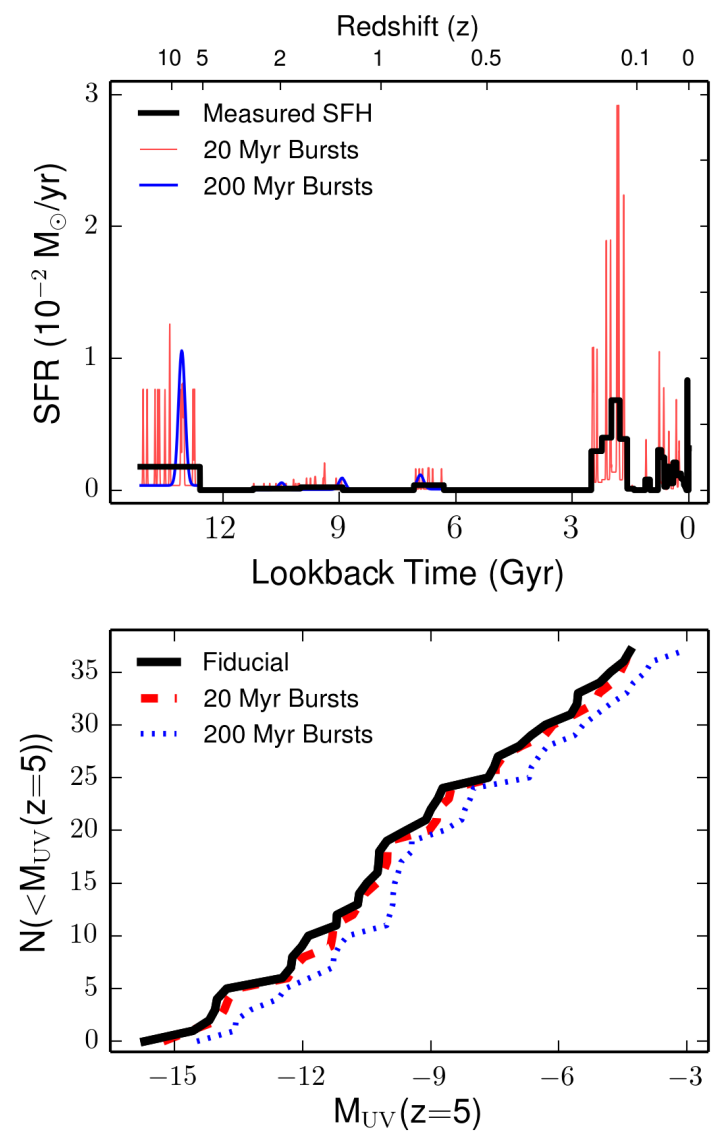

Figure 1. Translation of SFHs into UV LFs. Top panel: SFH vs. lookback time at the native time resolution (black) for a single galaxy. The red and blue lines illustrate the variations in intra-time bin bursts that we considered (see $\$ 2.2$ for details). Bottom panel: The cumulative $z=5 \mathrm{UV}$ LF for all 37 galaxies in the sample. The thick black line is the UV LF assuming the native time resolution of the SFHs. The red and blue lines illustrate the variation in the mean UV LF for model SFHs that include bursts on 20 and $200 \mathrm{Myr}$ timescales.

owing to lingering uncertainties in stellar evolution and the subtle changes in isochrones at old ages (e.g., Gallart et al. 2005).

\subsection{From Star Formation Histories to UV Fluxes}

Following the procedure detailed in Johnson et al. (2013), we use FSPS to translate each SFH into the GALEX restframe FUV flux as a function of time. As a first step, we correct for aperture effects inherent to the observations. The $H S T$ field-of-view typically covers a modest fraction of most galaxies $(\sim 5-50 \%)$, and thus HST-based SFHs are not necessarily globally representative. We derive an approximate aperture correction by comparing the synthesized $z=0 \mathrm{~V}$ band luminosity with the observed V-band luminosity from McConnachie (2012). We multiply each SFH by the ratio of these quantities and use the resulting re-scaled SFHs and FSPS to compute the FUV flux profile. When synthesizing the UV fluxes with FSPS, we assume a constant metallicity of $0.2 Z_{\odot}$ and no internal dust extinction. The latter assumption is reasonable as the UV spectral slopes of faint high redshift galaxies are consistent with a dust-free spectral energy distribution (e.g., Bouwens et al. 2012; Dunlop et al. 2013).

For lookback times older than a few Gyr, the native time bins of the SFHs are larger than the timescale of UV emission $(\sim 100 \mathrm{Myr})$. We must therefore explore the effects of
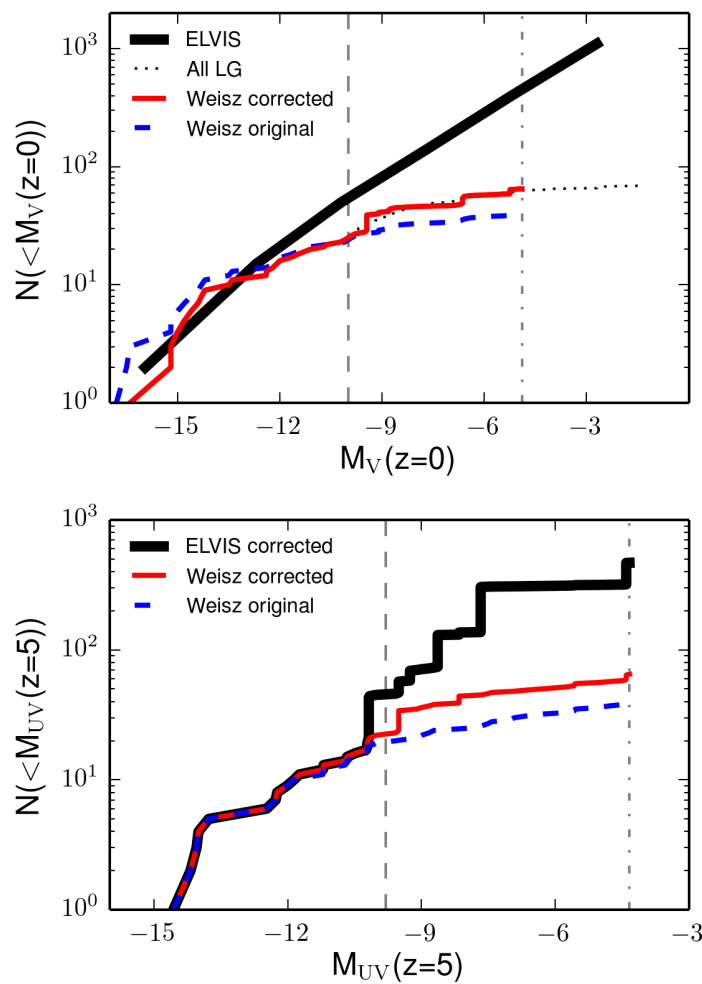

Figure 2. Illustration of completeness corrections applied to the dwarf galaxy data. Top panel: The $z=0 \mathrm{~V}$-band luminosity function of the original sample (blue), the sample corrected by environment (red), the entire LG dwarf LF (black dashed), and the predicted LF of the LG from the EVLIS simulation (black solid). The grey dot-dashed line indicates the faint limits of our LG data at $z=0$ and the dashed line indicates our conservative sample cut at $\mathrm{M}_{V}(z=0)=-10$. Bottom panel: Completeness corrections applied to the $z=5$ UV LF. Throughout the paper we use the ELVIS corrected UV LFs.

intra-bin star formation variation on the synthesized UV LFs. We quantify the amplitude of this effect by considering many permutations of stochastic intra-bin bursts. Two representative examples are shown in Figure 1. In both examples, $80 \%$ of the total stellar mass is formed in the burst phase (mass is, of course, conserved relative to the fiducial SFH), and the bursts have the same contrast amplitude of 20 relative to the 'off' burst star formation rate. The two models differ in burst timescales of 20 and $200 \mathrm{Myr}$ (red and blue lines, respectively). The $20 \mathrm{Myr}$ model is patterned after SFHs from fully cosmological simulations of dwarf galaxies (e.g., Governato et al. 2014), while the 200 Myr bursts illustrate fairly dramatic changes in the UV flux profile, as discussed in Weisz et al. (2012).

We assume these two burst models for all galaxies in our sample, and plot the resulting $z=5 \mathrm{UV}$ LFs in the bottom panel of Figure 1. For the purposes of this exploratory study, we largely mitigate the effects of intra-bin bursts by placing our UV LFs in 3-magnitude wide magnitude bins. This is also desirable as our sample only contains 37 galaxies, yet spans a wide range in UV luminosities.

We must also consider both random and systematic uncertainties in the native SFHs themselves. Random uncertainties are due to the finite number of stars in a CMD, and generally scale in amplitude with the sparsity of the observed CMD. Synthesizing the random uncertainties for all galaxies via a Monte Carlo analysis is computationally prohibitive. Instead, we analyze representative SFHs to place bounds on the random error range. From this exercise we conservatively adopt 
Table 1

Rest-Frame UV LFs of Local Group Dwarf Galaxies

\begin{tabular}{|c|c|c|}
\hline$M_{\mathrm{UV}}$ & & $\Phi_{*}\left(\mathrm{Mpc}^{-3} \mathrm{mag}^{-1}\right)$ \\
\hline \multicolumn{3}{|c|}{$z=0.75$} \\
\hline$-13.44 \pm 1.5$ & & $0.0134 \pm 0.0071$ \\
\hline$-10.44 \pm 1.5$ & & $0.0268 \pm 0.0142$ \\
\hline$-7.44 \pm 1.5$ & & $0.092 \pm 0.0416$ \\
\hline$-4.44 \pm 1.5$ & & $0.3513 \pm 0.2151$ \\
\hline$-1.44 \pm 1.5$ & & $0.2459 \pm 0.165$ \\
\hline \multicolumn{3}{|c|}{$z=1.25$} \\
\hline$-15.53 \pm 1.5$ & & $0.0083 \pm 0.0059$ \\
\hline$-12.53 \pm 1.5$ & & $0.0207 \pm 0.0155$ \\
\hline$-9.53 \pm 1.5$ & & $0.2653 \pm 0.1063$ \\
\hline$-6.53 \pm 1.5$ & & $0.0539 \pm 0.0244$ \\
\hline$-3.53 \pm 1.5$ & & $1.3885 \pm 1.2372$ \\
\hline \multicolumn{3}{|c|}{$z=2$} \\
\hline$-11.75 \pm 1.5$ & & $0.191 \pm 0.1342$ \\
\hline$-8.75 \pm 1.5$ & & $0.2834 \pm 0.1503$ \\
\hline$-5.75 \pm 1.5$ & & $0.9242 \pm 0.5239$ \\
\hline$-2.75 \pm 1.5$ & & $1.2877 \pm 0.7885$ \\
\hline \multicolumn{3}{|c|}{$z=3$} \\
\hline$-13.92 \pm 1.5$ & & $0.0333 \pm 0.0189$ \\
\hline$-10.92 \pm 1.5$ & & $0.0956 \pm 0.0454$ \\
\hline$-7.92 \pm 1.5$ & & $0.2162 \pm 0.0899$ \\
\hline$-4.92 \pm 1.5$ & & $1.4591 \pm 0.8935$ \\
\hline \multicolumn{3}{|c|}{$z=4$} \\
\hline$-13.82 \pm 1.5$ & & $0.3184 \pm 0.151$ \\
\hline$-10.82 \pm 1.5$ & & $1.8523 \pm 0.7706$ \\
\hline$-7.82 \pm 1.5$ & & $5.9041 \pm 2.6702$ \\
\hline$-4.82 \pm 1.5$ & & $4.5149 \pm 3.91$ \\
\hline \multicolumn{3}{|c|}{$z=5$} \\
\hline$-15.66 \pm 1.5$ & & $0.1608 \pm 0.1206$ \\
\hline$-12.16 \pm 1.5$ & & $1.1793 \pm 0.5334$ \\
\hline$-8.66 \pm 1.5$ & & $3.8774 \pm 1.454$ \\
\hline$-5.16 \pm 1.5$ & & $2.573 \pm 2.1117$ \\
\hline
\end{tabular}

a fractional uncertainty of $50 \%$ on the SFH of each galaxy.

Systematic uncertainties are due to inherent shortcomings with the stellar models used to measure the SFHs (e.g., Dolphin 2012). Their amplitude generally scales inversely as a function of photometric depth, i.e., deeper CMDs provide more secure leverage on the full SFH of a galaxy relative to a shallower CMD because they probe more age sensitive features and older main sequence turnoffs (MSTOs). Correctly synthesizing these systematic uncertainties is beyond the scope of this current exercise but, in principle, can be be done in future analyses.

Finally, we use a Kroupa (2001) IMF for our analysis, the same as adopted by high redshift studies. However, a variable IMF (e.g., Zaritsky et al. 2012; Geha et al. 2013) would systematically affect the shape of the UV LF, but modeling this effect is beyond the scope of this paper.

\subsection{From UV Fluxes to Luminosity Functions}

Having constructed UV LFs for our 37 galaxies, we now correct for two sources of incompleteness. First, we account for known LG dwarfs that are not included in our sample. The sample is split by environment into galaxies associated with the MW, M31, and field, which allows us to account for the different selection functions (owing to different galaxy discovery surveys) and different average SFHs across environments. Within each environment we weight the galaxies in our sample by the LF of that environment. The result of this correction is shown in Figure 2. Note that our sample only extends as faint $M_{\mathrm{V}}=-4.9$ at $z=0$, and we ignore any galaxies fainter than this limit.

We next correct for undetected galaxies expected to exist in the LG, either because of incomplete sky coverage or flux and/or surface brightness limits. While a number of empirical estimates of 'missing' galaxies exist for MW satellites (e.g., Koposov et al. 2008; Tollerud et al. 2008), there are no such calculations for the entire LG. Therefore, we use predictions made by the ELVIS N-body simulation of LG-like environments (Garrison-Kimmel et al. 2014) to estimate the number of expected low-mass galaxies located within the LG at $z=0$. As shown by the solid black line in the top panel of Figure 2, nearly an order of magnitude more faint systems are expected to exist than are currently known. In this correction process, we propagate the fractional Poisson errors per magnitude bin from our intrinsic $z=0 \mathrm{~V}$-band LF to the UV LFs at all redshifts.

We illustrate the effect of the various volume corrections on the $z=5$ UV LF in the bottom panel of Figure 2. Clearly, accounting for missing galaxies is the dominant correction factor.

The normalization of the LG LF need not be the same as the high redshift field galaxy LF, both because of differential evolution of the LG with respect to the field and because of sample variance due to large scale structure in both samples. We address this by including separate normalization terms when fitting Schechter functions to the LG and literature LFs. Specifically, we model the combination of our LG data and literature data with a Schechter function of the form

$$
\Phi(M)=0.4 \ln 10 \Phi_{*}\left(10^{-0.4\left(\mathrm{M}-\mathrm{M}_{*}\right)}\right)^{\alpha+1} e^{-10^{0.4\left(\mathrm{M}-\mathrm{M}_{*}\right)}},
$$

where $\Phi_{*}, \mathbf{M}_{*}$, and $\alpha$ follow the standard definitions of normalization, characteristic magnitude, and faint end power-law slope. At each redshift we fit the literature data and reconstructed LG LFs simultaneously and require $\mathrm{M}_{*}$ and $\alpha$ to be the same for both Schechter functions; only the normalizations are allowed to differ between the two datasets. We then write the log-likelihood function for the combined datasets as

$$
\begin{gathered}
\ln P_{\text {total }}=\ln P+\ln P^{\prime} \propto \\
-0.5\left(\sum_{i} \frac{\left(y_{\text {literature }, i}-\Phi_{i}\right)^{2}}{\sigma_{\text {literature }, i}^{2}}+\sum_{j} \frac{\left(y_{\mathrm{LG}, j}-\Phi_{j}^{\prime}\right)^{2}}{\sigma_{\mathrm{LG}, j}^{2}}\right),
\end{gathered}
$$

where $P$ and $P^{\prime}$ are Gaussian likelihood functions, $y$ and $\sigma$ are the UV luminosity values and uncertainties per magnitude bin from the literature and LG, respectively, and $\Phi$ and $\Phi^{\prime}$ are the Schechter functions for the literature and LG data. We place broad top-hat priors on each of the four parameters to construct a posterior probability distribution, $P\left(\Phi_{*}, \mathrm{M}_{*}, \alpha, \Phi_{*}^{\prime} \mid\right.$ $\left.\mathrm{y}_{\text {literature }}, \sigma_{\text {literature }}, \mathrm{y}_{\mathrm{LG}}, \sigma_{\mathrm{LG}}\right)$ and sample it using the ensemble affine-invariant Markov chain Monte Carlo routine emcee (Foreman-Mackey et al. 2013). At each redshift we first fit only the literature data in order to verify consistency in recovered parameters with published values, and then proceeded to analyze the joint literature and LG datasets.

\section{RESULTS \& DISCUSSION}

The main result of this Letter is shown in Figure 3, where we plot UV LFs from $z=0.75$ to $z=5$. Included in each panel are the reconstructed LFs based on LG dwarfs (grey), literature data points (open symbols), best fit Schechter functions from the literature (dashed lines), and the median Schechter function of the posterior distribution (thick colored lines) along with $68 \%$ confidence interval (shaded envelopes). As 


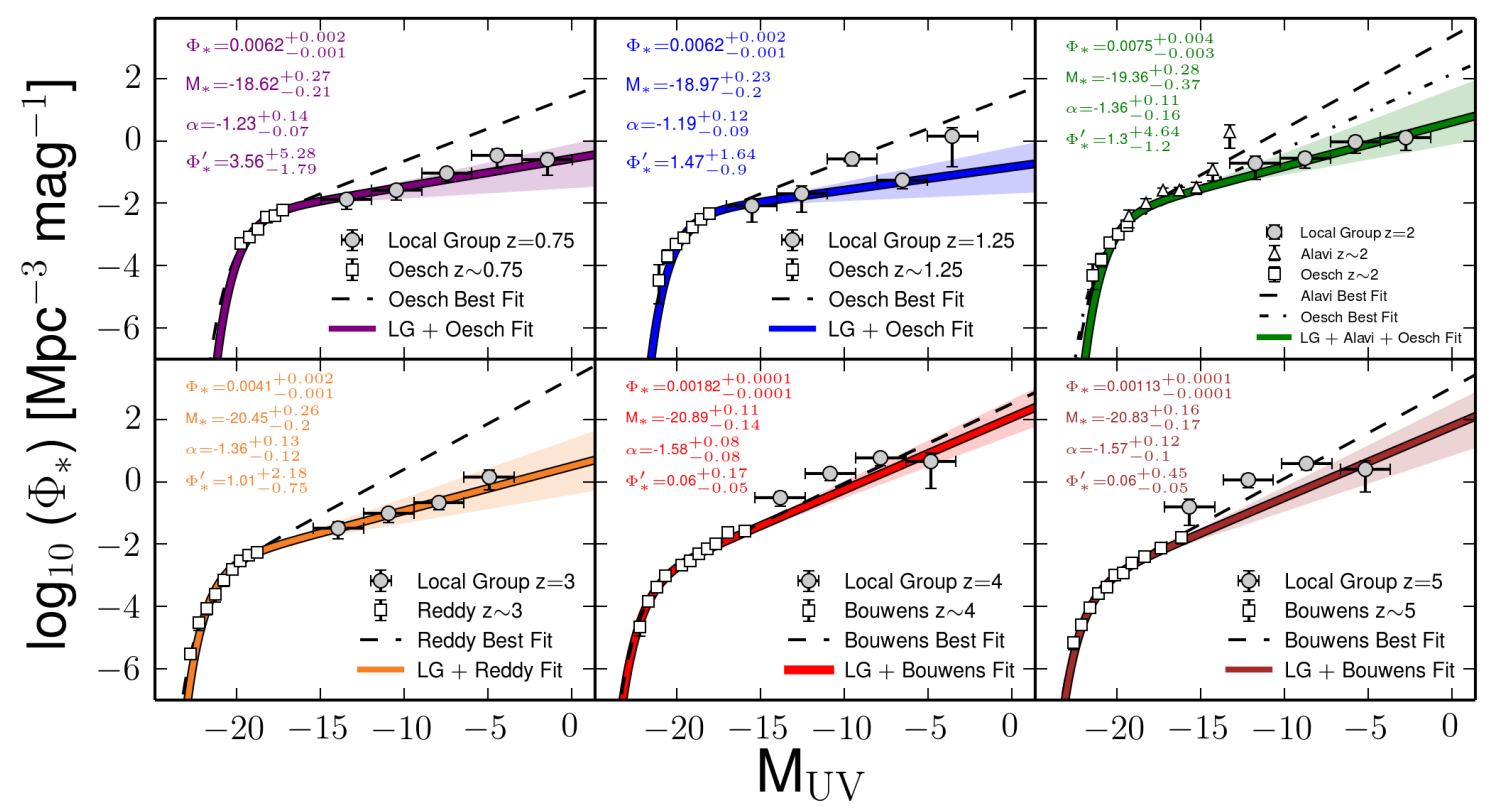

Figure 3. UV LFs at redshifts of $z=0.75,1.25,2,3,4$, and 5 . LG data are in grey and select literature values are shown as open symbols $(z \sim 0.75,1.25,2$ : Oesch et al. 2010; $z \sim 2$ : Alavi et al. 2014; $z \sim 3$ : Reddy \& Steidel 2009; $z \sim 4$, 5: Bouwens et al. 2014). The LG data has been normalized to the literature data at each redshift for plotting purposes only. The thick colored line represent the median Schechter function fit to the LG and literature data. The colored envelope enclose the $68 \%$ confidence interval. Best fit Schechter functions from the literature are shown as dashed/dot-dashed lines. Each panel also contains the 16th, 50th, and 84th percentiles for the Schechter function parameters.

discussed in $\$ 2.2$ only statistical uncertainties are included for the LG data. Our reconstructed UV LFs are listed in Table 1.

There are several important features in this Figure. First, the the LG dwarf data allow an estimate of the UV LF to extremely faint limits. At $z=2$ the fossil record extends the UV LF fainter by a factor of $\sim 10^{4}$ compared to the lensed observations of Alavi et al. (2014) and $\sim 10^{6}$ fainter than other existing data. Similar gains are seen at all redshifts, and are particularly notable at $z=0.75$, where the limits of the LG data extend down to a remarkable $M_{\mathrm{UV}} \sim-1.5$. Synthesizing UV fluxes for $z \lesssim 0.5$ becomes challenging because of the need to include uncertain contributions from ancient UV bright populations (e.g., post-AGB and blue horizontal branch stars).

A second notable feature is the shape of the LG data. Although we have made several relatively strong assumptions to construct these UV LFs, they exhibit a shape that is in good agreement with direct observations of the UV LF at higher luminosities. Perhaps most importantly, there is no evidence of a turnover in the UV LFs down to extremely faint limits the data are consistent with a single power-law slope at faint luminosities over a dynamic range of $\gtrsim 10^{4}$.

A key advantage of adding in the LG data is increased accuracy and precision in the recovery of Schechter parameters compared to using high luminosity data alone. As is clear from Figure 3, the direct measurements (Arnouts et al. 2005; Wyder et al. 2005; Sawicki \& Thompson 2006; Yoshida et al. 2006; Iwata et al. 2007; McLure et al. 2009; Ouchi et al. 2009; Reddy \& Steidel 2009; Hathi et al. 2010; Oesch et al. 2010; Bradley et al. 2012; Oesch et al. 2012; Sawicki 2012; Schenker et al. 2013; McLure et al. 2013; Alavi et al. 2014; Bouwens et al. 2014) of the UV LF generally only probe the bright end and the knee of the LF, which yields a significant amount of uncertainty in and degeneracy between the Schechter parameters.

Our resulting Schechter parameters are shown in Figure 4, along with literature values based only on the direct high red- shift measurements. In general, we find broad consistency with literature values of $\alpha$ at each redshift, although we highlight the significant uncertainties $(\sim 0.5 \mathrm{dex})$ in most literature values compared to this work. When comparing the other Schechter parameters, the agreement between our results and the literature is less impressive, especially at $z<2$. In this regime the direct measurements probe a limited dynamic range that results in large uncertainties and covariances between the Schechter parameters. Deeper rest-frame UV data at $0.5<z<1.5$ would be valuable in clarifying these apparent discrepancies.

In the bottom panel of Figure 4 we show the ratio of $\Phi_{*}^{\prime}$ and $\Phi_{*}$, the Schechter function normalizations for the LG and high redshift LFs, respectively. Their ratio encodes information regarding the relative behavior of the LG and high redshift LF. We would expect this ratio to be constant with redshift if the LG population was representative of the high redshift field population at each redshift. If instead the LG dwarfs have SFHs that systematically differ from the (higher luminosity) high redshift field population, then this ratio should change with redshift. This ratio might also evolve if the present LG population represents only a small fraction of the high redshift progenitor LG population. The uncertainties on this ratio are large and are broadly consistent with constant. Future improvements both in the high redshift data and the number of LG SFHs could significantly reduce these errors and would be valuable for placing the LG in a broader cosmological context.

There are several important assumptions and uncertainties in this analysis. As shown in Figure 1, the intra-bin SFH variation introduces modest uncertainties in the reconstructed UV LFs. This can be properly marginalized over in a more comprehensive analysis. A more important concern are systematic errors, which are typically dominant in the SFHs measured from CMDs that do not include the ancient MSTOs.

We have assumed that the LG dwarfs are dust-free and have 


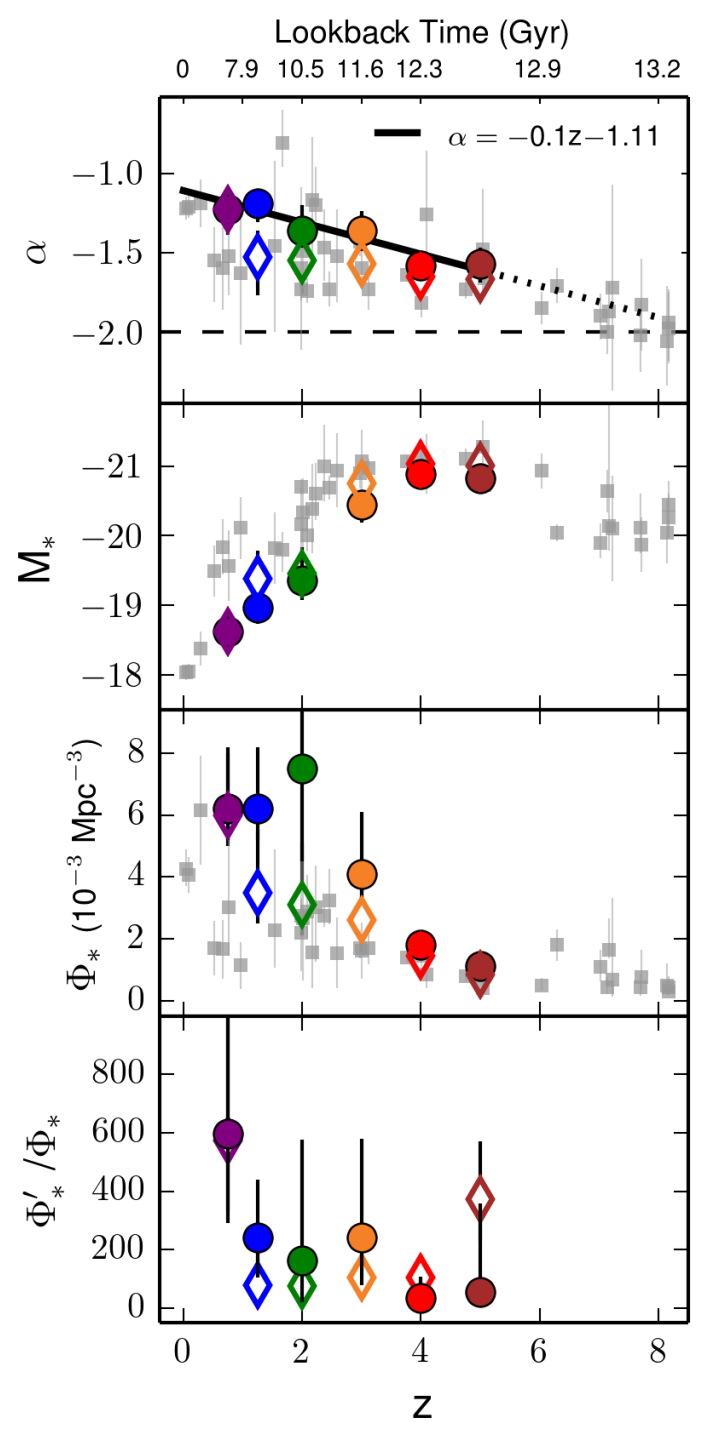

Figure 4. Derived Schechter parameters as a function of redshift. In each panel, the large circles are measurements from the present work, open diamonds are for results when using galaxies with $\mathrm{M}_{V}(z=0) \lesssim-10$, and the grey squares are literature data referenced in $\$ 3$. In the top panel, the solid black line reflects the $\alpha-z$ relationship measured from our data and the dotted line is an extrapolation to $z=8$. The bottom panel shows the ratio of the normalization parameter for the LG data and the direct high redshift measurements.

a single metallicity at higher redshift. This is a reasonable assumption given current observations, and it is worth noting that adding a constant amount of extinction to all the LG galaxies would only affect $\Phi_{*}^{\prime}$; in order to change $\alpha$ one would require a systematic variation of $A_{V}$ with dwarf galaxy luminosity.

Other limitations in the present study include the corrections required to estimate LFs from the 37 LG dwarfs. As shown in Figure 2, the incompleteness corrections at the faintest luminosities are a factor of $\sim 10$ and rely on $N$-body dark matter simulations (e.g., an assumed SHM relation). However, the corrections at brighter luminosities are quite small. If we restrict our analysis to luminosities where the LG data are believed to be highly complete, $\left(\mathrm{M}_{\mathrm{V}} \lesssim-10\right.$ at $\left.z=0\right)$, we recover consistent, but less precise, Schechter parameters at most redshifts (open symbols in Figure 4).

Finally, we expect that the present day LG population rep- resents only a fraction of the progenitor galaxy population owing to the disruption of dwarf galaxies over time (i.e., mostly accretion onto the MW/M31, but potentially dwarfdwarf mergers as well; Deason et al. 2014). If this disruption process is weakly dependent on the galaxy luminosity then our main results will be unaffected. We might expect a weak dependence because the correlation between dwarf galaxy light and total gravitational mass is very weak (e.g., Strigari et al. 2008), and it is the latter quantity that more directly controls the efficiency of tidal stripping and merging. It should be possible to estimate these effects directly with current $N$-body simulations.

These limitations and uncertainties can be addressed and reduced with upcoming facilities. Wide-field capabilities of the LSST will discover and better characterize the LG dwarf population, reducing the magnitude of volume and aperture corrections, and minimizing sample variance considerations (Robertson 2010). JWST and WFIRST will provide unprecedented depth to better characterize the brighter end of the high redshift LF and reach the oldest MSTOs of the dwarf galaxy populations in and beyond the LG.

Properly addressing these limitations will ensure that the reconstructed LFs of nearby dwarfs will play an important role in characterizing the faint galaxy population at high redshifts. Such systems are believed to play a critical part in reionization of the universe, but are too faint for direct detection in the foreseeable future. With our novel technique, we have demonstrated the existence of a large number of faint systems at high-redshifts. Their UV LFs show no hint of a turnover down to very faint limits, and suggest that current extrapolations of the faint-end slope to higher redshifts are justified.

The authors would like to thank the anonymous referee for detailed comments that helped improve the paper. We would also like thank Julianne Dalcanton for insightful discussion on the interpretation of $\Phi^{\prime}$, and Mike Boylan-Kolchin and James Bullock for general discussion of reionization and the LG. DRW is supported by NASA through Hubble Fellowship grant HST-HF-51331.01 awarded by the Space Telescope Science Institute. DRW was also partially supported in part by the National Science Foundation under Grant No. PHYS-1066293 and the hospitality of the Aspen Center for Physics, and by Hans-Walter Rix and the MPIA. CC is supported by Packard and Sloan Foundation Fellowships. This research made extensive use of NASA's Astrophysics Data System Bibliographic Services.

\section{REFERENCES}

Alavi, A., Siana, B., Richard, J., et al. 2014, ApJ, 780, 143

Arnouts, S., Schiminovich, D., Ilbert, O., et al. 2005, ApJ, 619, L43

Bouwens, R. J., et al. 2012, ApJ, 754, 83

Bouwens, R. J., Illingworth, G. D., Oesch, P. A., et al. 2014, ArXiv e-prints, arXiv: 1403.4295

Boylan-Kolchin, M., Bullock, J. S., \& Garrison-Kimmel, S. 2014, MNRAS, 443, L44

Bradley, L. D., Trenti, M., Oesch, P. A., et al. 2012, ApJ, 760, 108

Conroy, C., Gunn, J. E., \& White, M. 2009, ApJ, 699, 486

Conroy, C., White, M., \& Gunn, J. E. 2010, ApJ, 708, 58

Deason, A., Wetzel, A., \& Garrison-Kimmel, S. 2014, ArXiv e-prints, arXiv:1406.3344

Dijkstra, M., Haiman, Z., Rees, M. J., \& Weinberg, D. H. 2004, ApJ, 601, 666

Dolphin, A. E. 2002, MNRAS, 332, 91

—. 2012, ApJ, 751, 60

Dolphin, A. E., Saha, A., Skillman, E. D., et al. 2003, AJ, 126, 187

Dunlop, J. S., Rogers, A. B., McLure, R. J., et al. 2013, MNRAS, 432, 3520 
Foreman-Mackey, D., Hogg, D. W., Lang, D., \& Goodman, J. 2013, PASP, 125,306

Gallart, C., Zoccali, M., \& Aparicio, A. 2005, ARA\&A, 43, 387

Garrison-Kimmel, S., Boylan-Kolchin, M., Bullock, J. S., \& Kirby, E. N. 2014, ArXiv e-prints, arXiv:1404.5313

Geha, M., Brown, T. M., Tumlinson, J., et al. 2013, ApJ, 771, 29

Governato, F., Weisz, D., Pontzen, A., et al. 2014, ArXiv e-prints, arXiv: 1407.0022

Hathi, N. P., Ryan, Jr., R. E., Cohen, S. H., et al. 2010, ApJ, 720, 1708

Iwata, I., Ohta, K., Tamura, N., et al. 2007, MNRAS, 376, 1557

Johnson, B. D., Weisz, D. R., Dalcanton, J. J., et al. 2013, ApJ, 772, 8

Koposov, S., Belokurov, V., Evans, N. W., et al. 2008, ApJ, 686, 279

Kroupa, P. 2001, MNRAS, 322, 231

Kuhlen, M., \& Faucher-Giguère, C.-A. 2012, MNRAS, 423, 862

McConnachie, A. W. 2012, AJ, 144, 4

McLure, R. J., Cirasuolo, M., Dunlop, J. S., Foucaud, S., \& Almaini, O. 2009, MNRAS, 395, 2196

McLure, R. J., Dunlop, J. S., Bowler, R. A. A., et al. 2013, MNRAS, 432, 2696

Oesch, P. A., Bouwens, R. J., Carollo, C. M., et al. 2010, ApJ, 725, L150

Oesch, P. A., Bouwens, R. J., Illingworth, G. D., et al. 2012, ApJ, 759, 135
Ouchi, M., Mobasher, B., Shimasaku, K., et al. 2009, ApJ, 706, 1136

Reddy, N. A., \& Steidel, C. C. 2009, ApJ, 692, 778

Robertson, B. E. 2010, ApJ, 716, L229

Robertson, B. E., Furlanetto, S. R., Schneider, E., et al. 2013, ApJ, 768, 71

Sawicki, M. 2012, MNRAS, 421, 2187

Sawicki, M., \& Thompson, D. 2006, ApJ, 642, 653

Schechter, P. 1976, ApJ, 203, 297

Schenker, M. A., Robertson, B. E., Ellis, R. S., et al. 2013, ApJ, 768, 196

Strigari, L. E., Bullock, J. S., Kaplinghat, M., et al. 2008, Nature, 454, 1096

Tollerud, E. J., Bullock, J. S., Strigari, L. E., \& Willman, B. 2008, ApJ, 688, 277

Weisz, D. R., Dolphin, A. E., Skillman, E. D., et al. 2014, ArXiv e-prints, arXiv: 1404.7144

Weisz, D. R., Johnson, B. D., Johnson, L. C., et al. 2012, ApJ, 744, 44

Windhorst, R. A., Cohen, S. H., Jansen, R. A., Conselice, C., \& Yan, H. 2006, New Astronomy Review, 50, 113

Wyder, T. K., Treyer, M. A., Milliard, B., et al. 2005, ApJ, 619, L15

Yoshida, M., Shimasaku, K., Kashikawa, N., et al. 2006, ApJ, 653, 988

Zaritsky, D., Colucci, J. E., Pessev, P. M., Bernstein, R. A., \& Chandar, R. 2012, ApJ, 761, 93 